\title{
Kernos
}

Revue internationale et pluridisciplinaire de religion grecque antique

$21 \mid 2008$

Varia

\section{Pierre BRULÉ, La Grèce d'à côté. Réel et imaginaire en miroir en Grèce antique}

\section{André Motte}

Édition électronique
URL : https://journals.openedition.org/kernos/1710

DOI : 10.4000/kernos. 1710

ISSN : 2034-7871

\section{Éditeur}

Centre international d'étude de la religion grecque antique

\section{Édition imprimée}

Date de publication : 1 janvier 2008

Pagination : 357-359

ISSN : 0776-3824

Référence électronique

André Motte, "Pierre BRuLÉ, La Grèce d'à côté. Réel et imaginaire en miroir en Grèce antique », Kernos [En ligne], 21 | 2008, mis en ligne le 15 septembre 2011, consulté le 24 août 2022. URL : http:// journals.openedition.org/kernos/1710; DOI : https://doi.org/10.4000/kernos.1710 
sein des représentations grecques de la physiologie des humeurs et des souffles. Les représentations religieuses y trouvent leur place, sans solution de continuité avec les autres aspects du savoir partagé des Grecs sur ces questions².

Vinciane Pirenne-Delforge

(F.R.S.-FNRS - Université de Liège)

Pierre BRULÉ, La Grèce d'à côté. Réel et imaginaire en miroir en Grèce antique, Rennes, Presses Universitaires de Rennes, 2007. 1 vol. 16, $5 \times 24 \mathrm{~cm}, 542$ p. (coll. Histoire). ISBN : 978-2-7535-0495-0.

Cet historien de l'Antiquité (qu'on ne présente pas aux familiers de la religion grecque) rassemble ici une vingtaine d'études dont la plupart ont déjà été publiées entre 1992 et 2006, deux d'entre elles étant encore à paraître, une étant inédite, et qui sont réparties entre les sept parties de l'ouvrage correspondant à autant de thématiques distinctes; les intitulés de chacun des articles sont présentés en détail plus loin (p. 364). Une solide préface met en perspective les études réunies et les situe dans le parcours de l'historien. Qu'on ne se méprenne pas au sujet du titre principal : il n'annonce pas une Grèce qui serait marginale, mais bien une Grèce familière à l'auteur et dont la fréquentation assidue a été pour lui - il insiste - source d'un plaisir sans cesse renouvelé, tant est riche de questions stimulantes l'investigation des documents innombrables et divers qu'elle nous a légués. Disons-le d'emblée, ce plaisir, P.B. le rend bien à ses lecteurs, grâce au choix des sujets traités, - le sous-titre fait bien voir les deux pôles entre lesquels ils oscillent, - et grâce à la manière de les traiter, marquée certes par un constant souci de rigueur et par une attention accordée aux questions de méthode, mais aussi par des touches plus personnelles: des curiosités originales, des questions insolites, une ouverture à la démarche comparative quand le sujet s'y prête, une prédilection pour le concret, une vive sensibilité, une certaine façon de s'impliquer et un humour certain...

Représentatif donc d'une pensée et d'un style, cet ouvrage, on le devine déjà, est tout le contraire d'un assemblage réalisé à la sauvette : la sélection des articles et des thèmes, qui couvrent un très riche éventail, a été dûment réfléchie, les contributions ont fait l'objet d'une mise à jour, voire, pour certaines d'entre elles, de remaniements, des sous-titres fréquents en guident la lecture, une bibliographie riche de plus de six cents titres précède un très copieux index ainsi que la liste de la vingtaine de tableaux et de la dizaine de figures que comporte le livre, la présentation est à tous égards très soignée.

On devra bien se borner ici à épingler ce qui relève de la religion grecque. Parmi les sept thématiques retenues, il en est deux qui relèvent directement et entièrement de cette discipline, soit la cinquième, intitulée "Le culte », et la sixième, intitulée «Le polythéisme ». Mais la dernière partie ("Approches de la parenté ») fait place aussi, dans trois des quatre études qui la composent, à une exégèse approfondie d'épiclèses qui concernent le "paysage parental» de Zeus (Patrôios, Phratrios, Ktèsios, Herkeios, etc.). Peuvent également intéresser l'approche religieuse les sujets relevant de la mythologie qu'exploitent plusieurs chapitres (au passage, pointons ici les éclairages nouveaux que l'A. projette, sans prétendre lever toutes les énigmes, sur le mythe d'Europe et sur les origines du nom de notre continent). Dans la thématique vouée au genre, la figure de proue du premier article n'est autre qu'Artémis (Des osselets et des tambourins pour Artémis), si présente dans tout ce qui a trait au corps et à la vie des

${ }^{2}$ Deux détails mineurs : dans les Euménides, les Érinyes ne se voient pas promettre « un siège près de

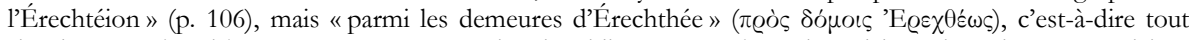
simplement «à Athènes »; quant au sanctuaire de Phlionte aux arbres duquel les prisonniers suspendaient leurs chaînes et qui ne comptait pas de statue, Pausanias l'attribue à Hèbè-Ganyméda et non à Héra (p. 173). 
filles. Et on pourrait citer encore d'autres études dans lesquelles affleurent des éléments religieux. Mais revenons-en aux deux rubriques principales sous ce rapport, à commencer par celle qui est vouée au culte et qui réunit trois contributions. Est reprise en premier lieu la belle étude sur les sacrifices et la procession des Panathénées (La cité en ses composantes), que les lecteurs de Kernos $\left(\mathrm{n}^{\circ}\right.$ 9, 1996) connaissent bien et qu'il suffit donc ici de rappeler. Une autre étude, qui est encore à paraître dans les actes de la IVe Celtic Conference in Classics et qui a été rédigée en collaboration avec Rachel Touzé, est consacrée aux êtres sacrifiés que sont les hiereia (ne pas traduire par "victime», est-il recommandé, vu les connotations très particulières qui s'attachent à ce mot et qui ne conviennent pas pour nos Grecs), aux qualités que les dieux et les hommes attendent d'eux (où l'on voit notamment que la psychè peut être fort proche du sôma) et à des particularités que parfois ils présentent (comme le fait de se désigner eux-mêmes pour le sacrifice), avec un curieux appendice montrant le «bœuf gras violé » qui, avant d'être conduit à l'abattoir, était mené en procession, au son des violes (d'où cette épithète insolite), dans les rues d'un petit village de la France profonde, le jour du mardi gras, en plein vingtième siècle... Non moins surprenantes sont certaines informations qu'apporte et commente l'A. dans son Héraclès à l'éprenve de la chèvre où, faute de pouvoir disserter longuement sur les rapports entre le héros civilisateur et les caprins, - rapports quasi inexistants sauf à les considérer en creux, - il nous offre en prime un véritable petit « essai de caprologie antique ». L'« être-au-monde » de la chèvre, comme il dit plaisamment, réserve d'agréables surprises. Saviez-vous que cet animal respire par les oreilles et manifeste une rare sensibilité au pneuma (à preuve le rôle décisif joué par une chèvre dans la découverte du site oraculaire de Delphes...), qu'il existe une espèce de poisson qui, amoureux de l'odeur que répandent les chèvres au bord de la mer, saute hors de l'eau pour s'en imbiber, que la chèvre a partie liée avec les origines du langage (il est du reste une fable où Ésope lui donne la parole...), ou encore que la pharmacopée gynécologique compte d'innombrables remèdes caprins? Ne pensez pas trop vite que tout cela n'a rien à voir avec la religion, car l'A. aura tôt fait de vous prouver que, dans ce genre d'histoire, "le surnaturel » peut être «très étroitement lié aux phénomènes naturels »; chassez le religieux, il revient au galop ! Il prend soin d'ailleurs de montrer aussi que les chèvres, volontiers assimilées aux filles, sont très présentes dans la mouvance artémisiaque, sans compter qu'Aphrodite apprécie pareillement les sacrifices auxquels elles participent. Cet intéressant petit traité témoigne en tout cas qu'un brin de tendresse pour ce petit herbivore sympathique n'est pas incompatible avec l'austère métier de l'historien; si vous en doutiez encore, rendez-vous à la page 265, où l'une des deux seules illustrations de l'ouvrage (sans compter les figures) est la photographie d'une chèvre qui vous observe du haut d'une falaise crétoise ainsi qu'à la jolie quatrième de couverture, où l'on discerne un paisible troupeau de chèvres assiégeant une automobile, l'une d'elles étant tout bonnement juchée sur le toit de la machine. N'est-ce pas là une parfaite illustration de «la Grèce d'à côté »?

La sixième partie de l'ouvrage procure une réflexion très substantielle sur le polythéisme. La première étude, qui porte sur les épiclèses (Le langage des épiclèses dans le polythéisme hellénique), a été présentée au colloque du CIERGA «Les panthéons des cités grecques » et est parue dans Kernos $(11,1998)$. On sait que le laboratoire du Crescam, que P.B. dirige, a mis depuis peu à la disposition du public une précieuse banque de données (BDDE) relative aux épiclèses divines (cf. Kernos 20, p. 217-228). Prolongeant la première enquête, la contribution suivante entend montrer le polythéisme en voie de transformation, grâce à l'examen des variations que subissent les listes des dieux invoqués dans les serments internationaux; des tableaux figurant en annexe fournissent ici un matériel impressionnant. L'A. s'attendait certes à faire apparaître ici des structures, mais c'est aussi « beaucoup d'histoire », observe-til, qu'il a trouvé. Le troisième texte est un inédit, issu d'une conférence faite à Liège en 2004. Contrairement à ses habitudes, l'historien délaisse cette fois les documents pour ne livrer 
que «de purs produits de pensoir». C’est à peine si, parlant en l'occurrence d'une « dérive », il ne s'en excuse pas! Quelle est pourtant la discipline qui ne gagne à ce que, de temps à autre, un peu de recul soit pris par rapport aux approches, aux démarches, aux procédures qui lui sont familières et qui sont héritées du fond des âges? P.B. commence par redire sa conviction que le polythéisme grec est déchiffrable, qu’il peut donc nous être intelligible, mais que nous nous y prenons sans doute mal pour qu'il en soit ainsi. Et d'esquisser rapidement plusieurs raisons pouvant expliquer cet état de choses. L'une d'elles, qui commande tout le développement qui suit, est l'habitude que nous avons de dissocier ce qui ne devrait pas l'être : un chapitre pour les dieux et les représentations que les Grecs s'en font, un chapitre pour les rites et les pratiques, alors que c'est dans une connexion étroite, dans la relation entre un acteur ou un groupe d'acteurs et un ou plusieurs destinataires imaginés que se vit le polythéisme, avec des actes appropriés qui ont besoin de tel medium, dans une série de circonstances données. Ces paramètres aux variantes innombrables sont successivement passés en revue et donnent lieu à une série de questions parfois dérangeantes. $\mathrm{Il}$ arrive que le parcours demande qu'on s'accroche, comme on dit, mais, sans qu'elles débouchent sur des conclusions révolutionnaires, nombre d'observations, de mises en garde et de recommandations paraissent bien salutaires.

Le but de ce trop bref compte rendu serait atteint s'il donnait le sentiment qu'en parcourant cet épais volume on apprend beaucoup, on s'étonne, on s'interroge, on réfléchit, on acquiesce ou on doute, et quelquefois on tique, fréquemment aussi on sourit, bref on prend bien du plaisir. Que souhaiter de plus?

André MOTTE

(Université de Liège)

BRENK Frederick E., With Unperfumed Voice. Studies in Plutarch, in Greek Literature, Religion and Philosophy, and in the New Testament Background, Stuttgart, Franz Steiner Verlag, 2007. 1 vol. $17 \times 24 \mathrm{~cm}, 544$ p. (Potsdamer Altertumswissenschaftliche Beiträge, 21). ISBN : 978-3-515-08929-6.

Avec ce troisième volume, F.E. Brenk poursuit le regroupement de sa production scientifique commencé, chez le même éditeur, sous les deux titres Religbting the Souls (1998) et Clothed in Purple Light (1999). Comme pour les deux précédents recueils, le titre emprunté à Plutarque, qui qualifie ainsi le style de la Pythie, annonce qu'en dépit de la diversité des champs d'investigation pratiqués par l'A., littératures grecque et latine, religion, philosophie, magie, judaïsme et christianisme originel, le centre d'intérêt principal du cofondateur de l'International Plutarch Society demeure Plutarque, un polygraphe et un polymathe dont l'œuvre immense oblige à explorer d'une manière rayonnante de nombreux secteurs du contexte culturel.

Trente et un textes, tous parus après 1999, sont donc réunis, dont vingt-sept articles, trois recensions de livres (deux monographies sur Plutarque et un recueil d'essais sur Dion de Pruse) et un obituaire consacré à Édouard Desplaces. Les articles, reproduits photographiquement, d'où une typographie quelque peu hétérogène, comprennent trois inédits, qui portent, l'un, sur Plutarque, et, les deux autres, sur Paul et la philosophie de son temps. Ils sont répartis entre cinq rubriques: Plutarch, Philosophy, Religion, Magic, et New Testament and Early Christianity.

La première de ces rubriques, de loin la plus fournie avec ses douze études, s'ouvre sur un inédit, "Uttering Unperfumed Words, Yet Reaches to a Thousand Years with Her Voice. Plutarch and His Age ", appuyé sur une bibliographie copieuse et bien à jour de dix pages. F.E.B. y propose très pertinemment de voir en Plutarque, non pas un historien, ni un philosophe 